\title{
Cellular kinetics of MED12-mutant uterine leiomyoma growth and regression in vivo
}

\author{
Vanida A Serna', Xin Wu', Wenan Qiang',3, Justin Thomas', Michael L Blumenfeld ${ }^{4}$ and Takeshi Kurita' \\ 1Department of Cancer Biology and Genetics, The Comprehensive Cancer Center, Ohio State University, Columbus, Ohio, USA \\ ${ }^{2}$ Center for Developmental Therapeutics, Chemistry of Life Processes Institute, Northwestern University, Evanston, Illinois, USA \\ ${ }^{3}$ Division of Reproductive Science in Medicine, Department of Obstetrics and Gynecology, Feinberg School of Medicine, Northwestern University, \\ Chicago, Illinois, USA \\ ${ }^{4}$ Department of Obstetrics and Gynecology, Ohio State University, Columbus, Ohio, USA
}

Correspondence should be addressed to T Kurita: takeshi.kurita@osumc.edu

\begin{abstract}
Cellular mechanisms of uterine leiomyoma (LM) formation have been studied primarily utilizing in vitro models. However, recent studies established that the cells growing in the primary cultures of MED12-mutant LM (MED12-LM) do not carry causal mutations. To improve the accuracy of LM research, we addressed the cellular mechanisms of LM growth and regression utilizing a patient-derived xenograft (PDX) model, which faithfully replicates the patient tumors in situ. The growth and maintenance of MED12LMs depend on 17 $\beta$-estradiol (E2) and progesterone (P4). We determined E2 and P4-activated MAPK and PI3K pathways in PDXs with upregulation of IGF1 and IGF2, suggesting that the hormone actions on MED12-LM are mediated by the IGF pathway. When hormones were removed, MED12-LM PDXs lost approximately $60 \%$ of volume within 3 days through reduction in cell size. However, in contrast to general belief, the survival of LM cells was independent of E2 and/or P4, and apoptosis was not involved in the tumor regression. Furthermore, it was postulated that abnormal collagen fibers promote the growth of LMs. However, collagen fibers of actively growing PDXs were well aligned. The disruption of collagen fibers, as found in human LM specimens, occurred only when the volume of PDXs had grown to over 20 times the volume of unstimulated PDXs, indicating disruption is the result of growth not the cause. Hence, this study revises generally accepted theories on the growth and regression of LMs.
\end{abstract}

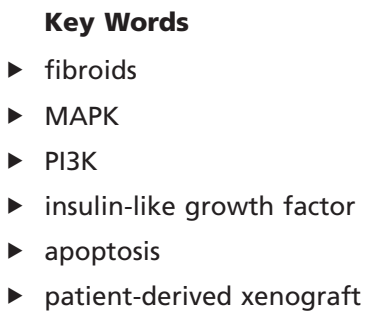

Endocrine-Related Cancer (2018) 25, 747-759

\section{Introduction}

Uterine leiomyoma (LM) is a benign smooth muscle tumor of the myometrium (MM) with an incidence of over 70\% of women of reproductive age (Marshall et al. 1997, Baird et al. 2003, Parker 2007). Approximately $25 \%$ of women of reproductive age require interventions due to symptoms of this tumor, which include heavy menstrual bleeding, pelvic pressure and pain (Myers et al. 2005). The epidemiology of LM reflects the hormone-dependent nature of this neoplasm: symptomatic LMs occur mostly during the ages of 30-40 years; there has not been a single case of LM reported in prepubescent girls; symptoms diminish by the time of menopause with volume reduction, but the symptoms may continue in menopausal women receiving hormone replacement therapies (Sener et al. 1996, Palomba et al. 2001, Yang et al. 2002). Accordingly, 
the gonadotropin-releasing hormone agonists, which induce hypogonadism, have been effectively used to suppress symptoms of LMs (Hodgson et al. 2017).

$17 \beta$-estradiol (E2) was considered to be the primary growth promoter of LMs for decades rather than progesterone (P4), based on experimental evidence from rodent and cell culture models as reviewed in our previous papers (Ishikawa et al. 2010, Wu et al. 2017). Meanwhile, clinical observations suggested the critical role of P4 in the pathogenesis of LMs: Labeling indices of LMs for proliferation markers were highest at the secretory phase, when the circulating P4 level is elevated (Tiltman 1985, Kawaguchi et al. 1989, Lamminen et al. 1992); proliferation indices of LMs in postmenopausal women increased significantly with combined estrogen plus progestin replacement but not with estrogen alone replacement (Lamminen et al. 1992) and selective progesterone receptor modulators reduced tumor size in clinical trials (reviewed in Murji et al. 2017). The direct evidence for the mitogenic effect of P4 on LMs was demonstrated in 2010 by a study with the patient-derived xenograft (PDX) model (Ishikawa et al. 2010): Subrenal grafts of human LM tissues in immunodeficient mice (Serna \& Kurita 2018) grew only when the host mice were supplemented with E2 and P4, but not E2 or P4 alone (Ishikawa et al. 2010, Qiang et al. 2014, Wang et al. 2014).

Recent comprehensive genome analyses identified four major LM subtypes with unique genetic alterations (Mehine et al. 2013, 2016), namely, MED12 mutant (MED12-LM) (Mäkinenetal.2011), HMGA2-overexpressing (HMGA2-LM) (Markowski et al. 2012, Bertsch et al. 2014, Mehine et al. 2016), fumarate hydratase (FH)-deficient and COL4A5/6-deletion subtypes. In addition, there were cases without any of these genetic alterations (Mehine et al. 2013, 2016). Our recent PDX study demonstrated that the two most prevalent LM subtypes, MED12-LM and HMGA2-LM subtypes, require E2 and P4 for growth (Wu et al. 2017). Nevertheless, it still remains unclear if all LM subtypes require E2 and P4 for growth and maintenance. In this current study, we exclusively focus on cellular kinetics of MED12-LM in regression and growth because this subtype accounts for approximately 70\% of all LM cases (Mäkinen et al. 2011), and its growth control by $\mathrm{E} 2+\mathrm{P} 4$ has been established.

Because it is widely believed that LM cells require steroid hormones for survival, the shrinkage and regrowth of LMs are purportedly caused by loss of cells through apoptosis and compensation of lost cells through the proliferation of hormone-deficiency-resistant stem cells, respectively (Maruo et al. 2000, Bulun et al. 2015).
Still, the hormone-dependent survival of LM cells has not been experimentally demonstrated. Moreover, growth and regression of LM have been predominantly studied utilizing cell culture models. However, recent studies have established that the primary cultures of MED12-LMs primarily consist of tumor-associated fibroblasts (TAFs) (Markowski et al. 2014, Bloch et al. 2017, Wu et al. 2017) rather than the mutation-bearing smooth muscle cells (SMCs). Accordingly, we investigated the cellular mechanism and signaling pathways involved in the growth, maintenance and regression of MED12-LMs utilizing a PDX model. Additionally, we investigated the signaling pathways involved in the actions of E2 and P4 in MED12-LM, focusing on the IGF-IGF1R pathway. While altered expression of IGF pathway molecules have been detected in LM samples (Yu et al. 2008, Baird et al. 2009, Peng et al. 2009), the functions and regulation of the IGF pathway in LMs were studied primarily in cell culture. Hence, in this study, we assessed the hormonal regulation of IGF pathway molecules in LM SMCs and TAFs utilizing the PDX model.

\section{Materials and methods}

\section{Preparation of MED12-LM and MM cells}

The acquisition and research use of surgical specimens were approved by the Institutional Review Board of the Ohio State University and Northwestern University and were conducted in accordance with the Belmont Report. The protocols for LM cell isolation and preparation of PDX have been described previously (Serna \& Kurita 2018). Briefly, fresh surgical specimens of human MM or LM tissues were enzymatically disassociated into single cells. Isolated cells were cultured for 3 days before preparation of xenografts, and each xenograft was prepared to contain $5 \times 10^{5}$ cells. The subtypes of LMs were examined with MED12 exon 2 sequencing and HMGA2 immunostaining in the original LM tissues as previously reported $(\mathrm{Wu}$ et al. 2017). Additionally, the presence of original MED12 mutations was confirmed in cultured cells from which PDXs were prepared. All MED12-LMs were confirmed to be positive for $\mathrm{FH}$ protein by immunostaining (not shown).

\section{Mice and PDX model}

All procedures involving animals in this study were approved by Northwestern University and The Ohio State University Animal Care and Use Committees. Mice were 
housed in a room designated for immunocompromised mice with sterile barrier cages provided food and water ad libitum. Temperature, humidity and photoperiod (14 light: 10 darkness) were kept constant. The details of the PDX procedure have been described previously (Serna $\&$ Kurita 2018). Briefly, mice were anesthetized with $90 \mathrm{mg} / \mathrm{kg}$ ketamine and $8 \mathrm{mg} / \mathrm{kg}$ xylazine, respectively, via i.p. injection. Grafts were surgically implanted under the subrenal capsule of kidneys in adult (aged 6-10 weeks) female NOD-scid Il2rg-null mouse hosts (Jackson Laboratory, Bar Harbor, ME, USA). All hosts were ovariectomized and supplemented with $70 \mathrm{mg}$ $\mathrm{E} 2+\mathrm{P} 4$ pellets subcutaneously. Mice were also treated with subcutaneous injections of $2.0 \mathrm{mg} / \mathrm{kg} /$ day meloxicam daily for $72 \mathrm{~h}$. Hormone pellets were replaced every 2 months under full anesthetic depth. Blood was collected by enucleation under full anesthetic depth, and plasma E2 and P4 levels were measured at The Ligand Assay and Analysis Core Facility, University of Virginia. The treatment schedule of the experiment is described in each section.

\section{Immunofluorescence and TUNEL assay}

The expression of proteins and activation of signaling pathways were analyzed by immunofluorescence (IF) assay for the following reasons: i) PDX consists of multiple cell types but the information about protein distribution among cell types is lost by immunoblotting and ii) since the size of PDX, particularly in the absence of hormones, is very small $\left(\sim 0.2 \mathrm{~mm}^{3}\right)$, multiple ( 10) PDXs are required for a single immunoblotting. IF was performed on $\geq 4$ samples per group from $\geq 2$ PDX experiments. Images were captured on a fluorescence microscope (BZ-9000; Keyence, Osaka, Japan) under identical conditions between samples for each antibody (Terakawa et al. 2016). The contrast of images was adjusted applying identical parameters to the images for each antibody by the batchprocess function of Adobe Photoshop CS6 (Adobe). Since the results are essentially identical between experiments, all IF images in the figures represent a PDX study with a c.130G >A MED12-LM.

The following primary antibodies were used at indicated dilutions: anti-phospho-ERK1/2 (MAPK3/1) (phopho-T202/Y201, 1:50, \#4370), anti-phosphoAKT (phopho-S473, 1:100, \#9271), anti-phospho-IRS1 (phosphor-Ser 636/639, 1:50, \#2388), anti-IGF1R $\beta$ (1:1000, \#3027) anti-human PECAM1 (1:100, \#3528) and anti-vimentin (1:200, \#9856) antibodies from Cell Signaling Technologies; anti-MKI67 (1:100, ab92742), anti- $\alpha$ SMA (ACTA2) (1:500, ab7817), anti-PECAM1 (1:200, ab2834), anti-IGF1 (1:1000, ab40657) and anti-IGF2 (1:200, ab9574) antibodies from Abcam; anti-MED12 (1:50, HPA003184; Sigma-Aldrich), anti-VWF (1:200, ECM590-21540; MilliporeSigma), anti-PECAM1 (1:200, ECM590-90214, MilliporeSigma), anti-FH (1:200, 109661-AP; Proteintech, Chicago, IL, USA), anti-ESR1 (1:100 RM9101-S, Lab Vision) and anti-PGR (1:200, A0098, Agilent Technologies) antibodies. Although the antiPECAM1 (ab2834) antibody recognizes both human and mouse PECAM1, it required a higher concentration (1:50) to detect human endothelial cells with our protocol. Thus, we refer this antibody as to anti-mouse PECAM1 hereafter. The following donkey polyclonal secondary antibodies (Jackson ImmunoResearch) were used at 1:800-1:1000 dilutions: Alexa-Fluor594 anti-mouse IgG $(\mathrm{H}+\mathrm{L})$ (715-586-150), Alexa-Fluor488 anti-rabbit IgG $(\mathrm{H}+\mathrm{L})$ (711-546-152), Alexa-Fluor594 anti-rabbit IgG $(\mathrm{H}+\mathrm{L})$ (711-586-152), biotinylated anti-rabbit IgG $(\mathrm{H}+\mathrm{L})$ (711-066-152) and biotinylated anti-rat IgG (H+L) (712-066-153). Biotinylated antibodies were used in conjunction with streptavidin-horseradish peroxidase (016-030-084, Jackson ImmunoResearch) and AlexaFluor488 conjugated streptavidin (1:500, S1123, Thermo Fisher Scientific). Bisbenzimide H 33258 (Hoechst) (1:10,000, Sigma-Aldrich) was used for nuclear staining in IF assay. Apoptosis was detected with DeadEnd Fluorometric TUNEL System (Promega) following the manufacturer's instruction. After rTdT enzyme treatment step, sections were incubated with anti-ACTA2 antibody overnight followed by incubation with Alexa-Fluor594 anti-mouse IgG (H+L) (1:1000, 715-586-150, Jackson ImmunoResearch) for $1 \mathrm{~h}$.

\section{Morphometric analysis}

The details of analysis have been described in previous papers (Ishikawa et al. 2010, Wu et al. 2017, Serna \& Kurita 2018). Briefly, SMCs in xenografts were identified with IF staining for ACTA2. MKI67 and TUNEL labeling indices were determined by counting MKI67 and TUNEL-positive cells among $\geq 687$ SMCs (ACTA2-positive cells) per xenograft, and the value of each graft was considered as an independent measurement. Statistical analyses were performed with the value of 3-8 xenografts per group. For the measurement of SMC size, SMC cytoplasm and SMC nucleus were selected by adjusting the threshold in red and blue (Hoechest) channels, respectively. The total SMC areas were measured in the image in which two channels 
were merged. SMC cell size $=$ total $\mathrm{SMC}$ area $\div$ SMC nuclear number.

\section{Quantitation of IF signals}

The target proteins were detected in the green channel, and the red channel was always used for ACTA2. IF images were captured utilizing Keyence BZ-9000 microscope at $40 \times$ magnification ( $\geq 2$ frames per sample) with parameters (e.g. exposure, gain, etc.) fixed for each antibody. In each image, ATCA2-positive pixels were selected by adjusting the threshold, and the average intensity of green signals (range from 0 to 255) in ACTA2-positive pixels was measured utilizing Image (NIH). In the same image, the ACTA2-positive area and the number of SMC nuclei (nucleus in the ACTA2-positive area) were also measured. The level of proteins per SMC was calculated as follows: Average intensity of green signals in ACTA2-positive pixels $\times$ ACTA2-positive pixel number $\div$ SMC nuclear number. The value of each PDX was normalized to the mean value of no-hormone group.

\section{Transmission electron microscopy}

MED12-LM PDXs from three independent experiments were analyzed. Glutaraldehyde-fixed fresh MED12-LM PDXs were diced into $1 \mathrm{~mm}^{3}$ cubes, rinsed in distilled water, transferred into $1 \%$ aqueous osmium tetraoxide and embedded in TAAB Emix resin (TAAB Laboratories Ltd, Aldermaston, Berkshire, UK). 0.6 $\mu \mathrm{m}$ sections were cut, mounted on glass slides and stained in 1\% aqueous toluidine blue in $1 \%$ sodium tetraborate for $30 \mathrm{~s}$ at $140^{\circ} \mathrm{C}$. They were then sectioned with a diamond knife in a Leica Ultracut E Ultramicrotome (Leica Microsystems, Wetzlar, Germany) with interference color gold of approximately $95 \mathrm{~nm}$. Sections were stained by immersion for $3 \mathrm{~min}$ in 99\% alcoholic saturated uranyl water, 3 min in Reynold's lead citrate and three washes in distilled water. Pictures were taken using a FEI Tecnai G2 Spirit transmission electron microscope (FEI, Hillsboro, Oregon, USA) at the Campus Microscopy and Imaging Facility, The Ohio State University.

\section{Statistical analysis}

The value of each xenograft was considered as single measurement, and the experimental data were presented as mean values with SDs. For statistical analysis, oneway ANOVA with post hoc Tukey's HSD test was used.
Statistical significance was indicated as: ns, nonsignificant; ${ }^{*} P<0.05,{ }^{*} P<0.01$ and ${ }^{* *} P<0.001$.

\section{Results}

\section{Cellular kinetics of LM regression}

We have previously demonstrated that PDXs of LM shrunk when E2 and/or P4 were withdrawn (Ishikawa et al. 2010, Qiang et al. 2014, Wu et al. 2017). In these studies, however, the response of xenografts was assessed only at $\geq 2$ weeks post hormone withdrawal, and thus, the immediate response of PDXs to hormone withdrawal was not addressed. Accordingly, we analyzed the cellular changes in MED12-LM (c.130G>C) PDXs at before (day 0) and 3 and 7 days after hormone withdrawal (Fig. 1A). A dramatic reduction in the volume of PDXs occurred within 3 days, and the volume changes in the following 4 days were relatively small (Fig. 1B). This pattern paralleled SMC size (Fig. 1C), which was reduced by $60 \%$ within 3 days, indicating SMC volume reduction is the primary factor contributing to the shrinkage of LMs. Meanwhile, the MKI67 labeling index in SMCs did not reach basal level until 7 days after hormone withdrawal (Fig. 1D), revealing proliferation activity remains longer than the signaling activity that maintains cell size. Despite the general belief that the loss of ovarian steroids triggers massive apoptosis in hormone-dependent tumors, apoptosis in LM SMCs (Fig. 1E) and non-SMCs remained at basal level $(<0.3 \%)$ during the rapid shrinkage of PDXs. TUNEL-positive cells were rare and often observed in small clusters (Fig. 1A), suggesting that apoptosis is likely triggered by the microenvironment rather than E2 and P4 directly.

\section{Survival of LM cells is hormone independent}

To assess the effect of E2 and P4 on MED12-LMs further, PDXs of two independent MED12-LMs were subjected to hormonal manipulations as shown in Fig. 2A. As the serum E2 and P4 dropped to basal levels (Fig. 2B), PDXs shrunk to $\sim 25 \%$ of original volume within 2 weeks after pellet removal (Fig. 2C and D). The volume of PDXs was further reduced after 4 more weeks without hormone supplements (Fig. 2D, no hormone (NH) at 10 weeks). This shrinkage was due to the reduction in SMC size (Fig. 2E), and apoptosis was undetectable in PDXs of the NH group at 6 and 10 weeks (not shown). Interestingly, E2 treatment attenuated the shrinkage of PDXs, as seen in a comparison of $\mathrm{NH}$ and E2 groups at 10 weeks in Fig. 2C, D and E. This indicates that E2 has P4-independent functions in 


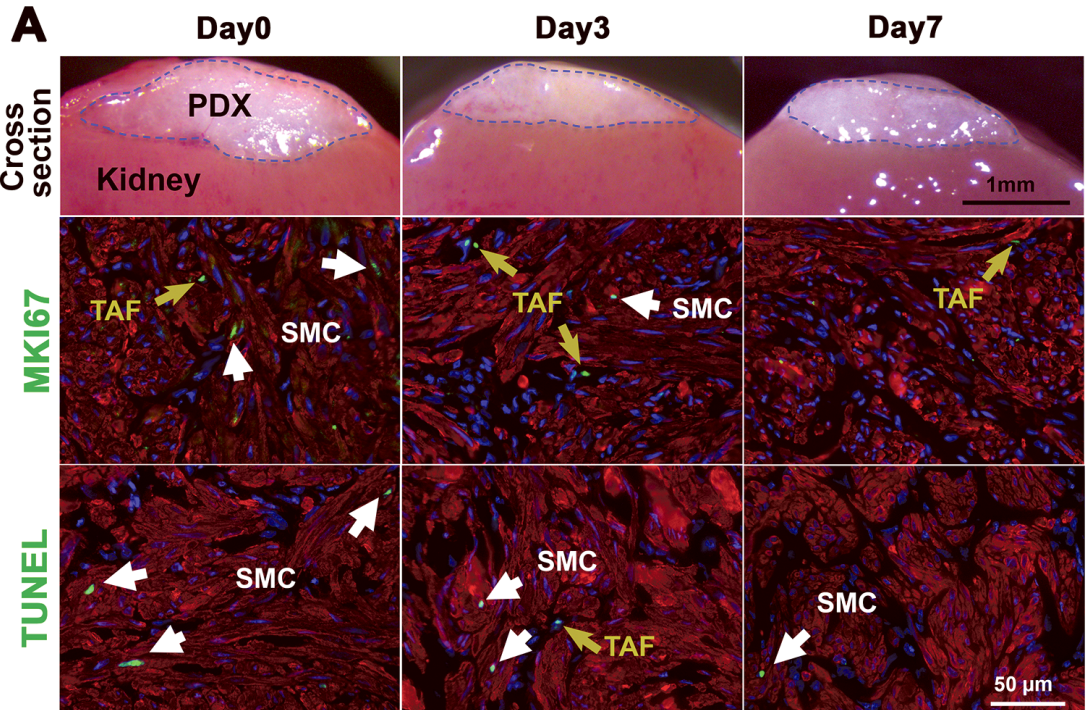

\section{MKI67 labeling index}

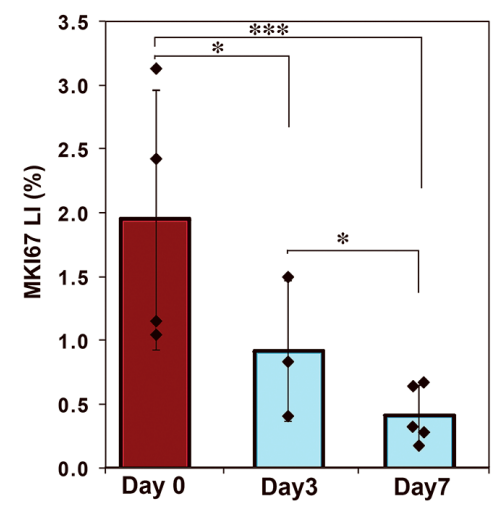

\section{B Tumor volume}

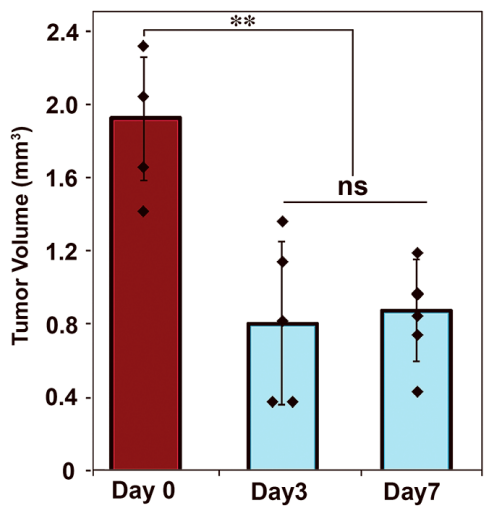

C SMC size

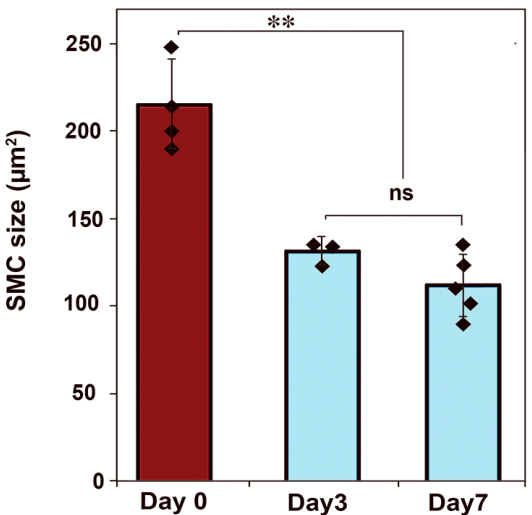

E TUNEL assay

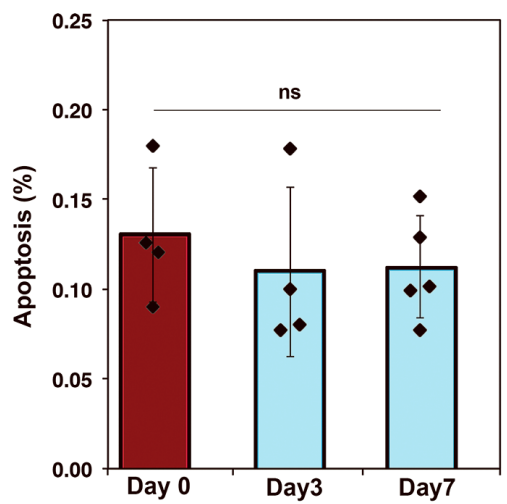

Figure 1

Cellular kinetics of LM PDX regression following hormone withdrawal - I: Short-term response. MED12-LM PDXs were grown for 4 weeks with E2+P4, and then the hormone pellet was removed (day 0). (A) Cross section of PDXs on the host kidney, MKI67 (green) and TUNEL (green) assays in PDXs at 0, 3 and 7 days after hormone withdrawal. SMCs are highlighted by ACTA2 (red) and nuclei are stained with Hochest (blue). (B) Volume of PDX. (C) Size (area) of SMCs by morphometric analysis. (D) MKI67 labeling index of SMCs. (E) Apoptotic rate by TUNEL assay. (E) Double staining images for TUNEL assay (green) and ACTA2 IF (red), apoptotic cells are indicated by arrows. The columns and error bars indicate average and SD, respectively. Each plot represents a measurement of a single PDX. The statistical significance by Tukey's HSD Test was indicated as: ns, non-significant; * $P<0.1$; $* * P<0.05$; and $* * * P<0.01$. LM, leiomyoma; PDX, patient-derived xenograft; SMC, smooth muscle cells; TUNEL, terminal deoxyribonucleotidyl-transferase nick-end labeling.

MED12-LMs, in agreement with our previous observation that E2 stimulates proliferation of TAFs. Nevertheless, the growth of PDXs was stimulated only when both E2 and P4 were re-supplemented, but not E2 alone (Fig. 2C and D).

\section{Regulation of MAPK and PI3K pathways by hormones}

The RAS-MAPK (MAPK) pathway and the phosphoinositide 3-kinase (PI3K)-AKT-mTOR (PI3K) pathway are two principal mechanisms through which extracellular cues control fundamental functions of cells such as survival, proliferation and metabolism. While the roles of MAPK and PI3K in LMs have been demonstrated in vitro, the accuracy of conclusions from cell culture studies is in question, as primary cultures of LMs primarily consist of TAFs. In addition, most in vitro studies are performed with LMs of unknown subtype, although the distinctive biology of subtypes has been demonstrated by recent studies and should be taken into account (Holdsworth-Carson et al. 2016, Mehine et al. 2016, Makinen et al. 2017, Wu et al. 2017). Therefore, we assessed how the activities of these pathways are regulated by $\mathrm{E} 2$ and $\mathrm{P} 4$ during the regression and regrowth of MED12-LM PDXs. MAPK pathway 
A
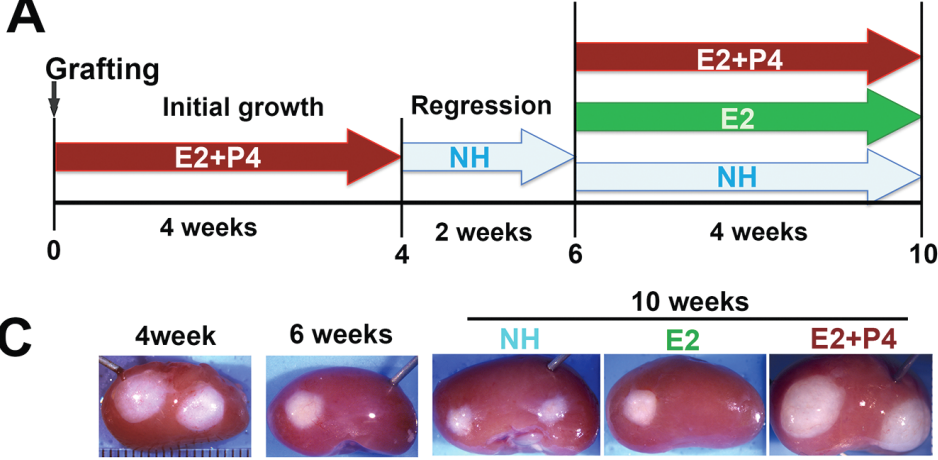

D

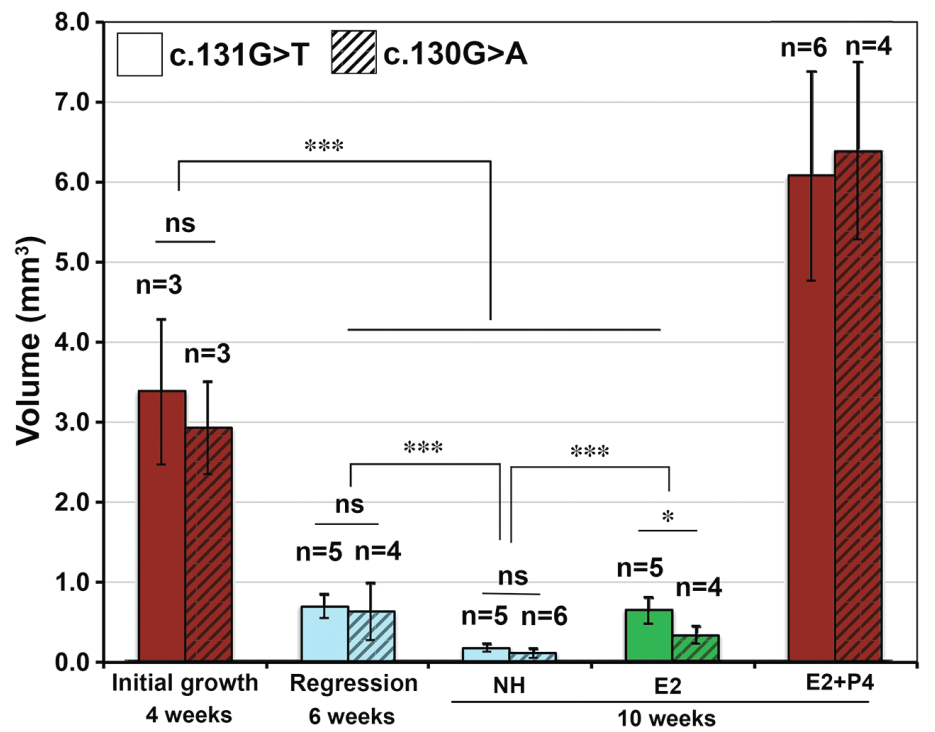

B
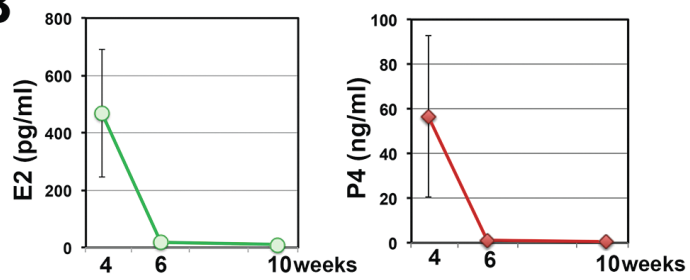

E

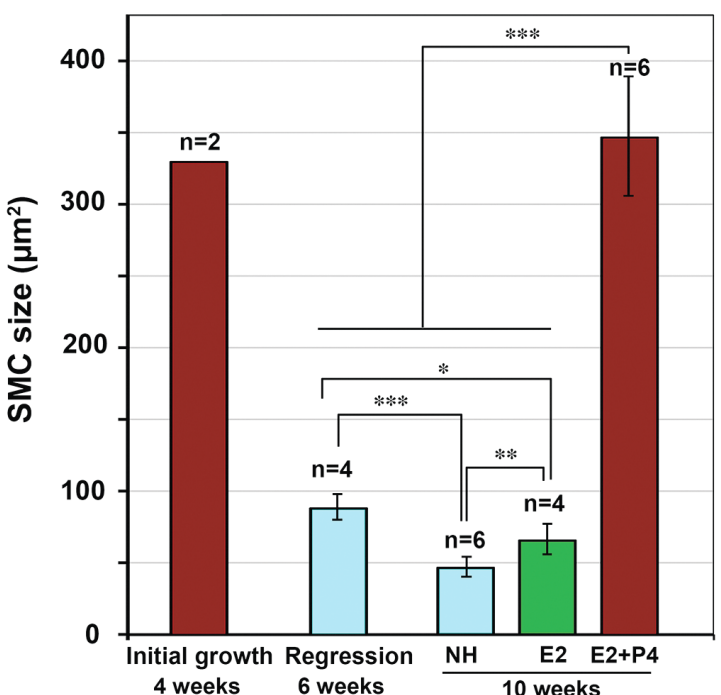

Figure 2

Cellular kinetics of LM PDX regression following hormone withdrawal - II: Long-term response. (A) Treatment schedule: PDXs were grown for 4 weeks with E2+P4, and then hormone pellets were removed. After 2 weeks of regression, host mice were supplemented with $\mathrm{NH}$, E2 or E2+P4 for 4 more weeks. (B) Average serum E2 and P4 levels. Error bars=s.D. $(n=5-12)$. (C) Gross appearance of PDXs on the host kidney. (D) Volume of PDX. The values of two independent studies are presented separately. (E) SMC size of PDXs from two independent studies. Equal number of PDXs from each experiment was included in the analysis. The columns and error bars indicate average and SD, respectively. The statistical significance by Tukey's HSD Test was indicated as: ns, non-significant; ${ }^{*} P<0.1 ; * * P<0.05$; and $* * * P<0.01$. NH, no hormone; PDX, patient-derived xenograft; SMC, smooth muscle cells. A full color version of this figure is available at https://doi.org/10.1530/ERC-18-0184.

activity was detected by phosphorylation of MAPK1 and MAPK3 (MAPK1/3) and MAPK/CDK substrate (MAPK-sub), and the activity of PI3K pathway was assessed by phosphorylation of AKT and ribosomal protein S6 (RPS6) as well as the nuclear exclusion of Forkhead box protein O1 (FOXO1). SMCs and TAFs retained substantial levels of MAPK and PI3K pathway activities even at 2 weeks after hormone withdrawal (6 weeks, Fig. 3A and B). Since the serum hormone concentrations already reached the basal levels (Fig. 2B), MAPK and PI3K pathway activities were likely maintained by local factors that persist for an extended period without E2 and P4. As the PDXs were further reduced in volume without hormones in the following 4 weeks, the activities of MAPK and PI3K pathways in SMCs eventually diminished (Fig. 3A and B). Nevertheless, basal levels of MAPK and PI3K activities were still detectable even 6 weeks after hormone withdrawal (NH 10 weeks), indicating that the viability of LM cells is independent of $\mathrm{E} 2$ and $\mathrm{P} 4$.

When the growth of PDXs was stimulated by re-supplementation of E2 and P4, the phosphorylation of MAPK1/3 and AKT significantly increased in SMCs (Fig. 3, white arrows). In contrast, the MAPK and PI3K pathway activities in TAFs were sustained at relatively constant levels irrespective of hormonal conditions (Fig. 3, yellow arrows).

Although the effect of E2 alone on the phosphorylation of MAPK1/3 and AKT was not readily recognized,
C) 2018 Society for Endocrinology Published by Bioscientifica Ltd. Printed in Great Britain 


\section{0 weeks}
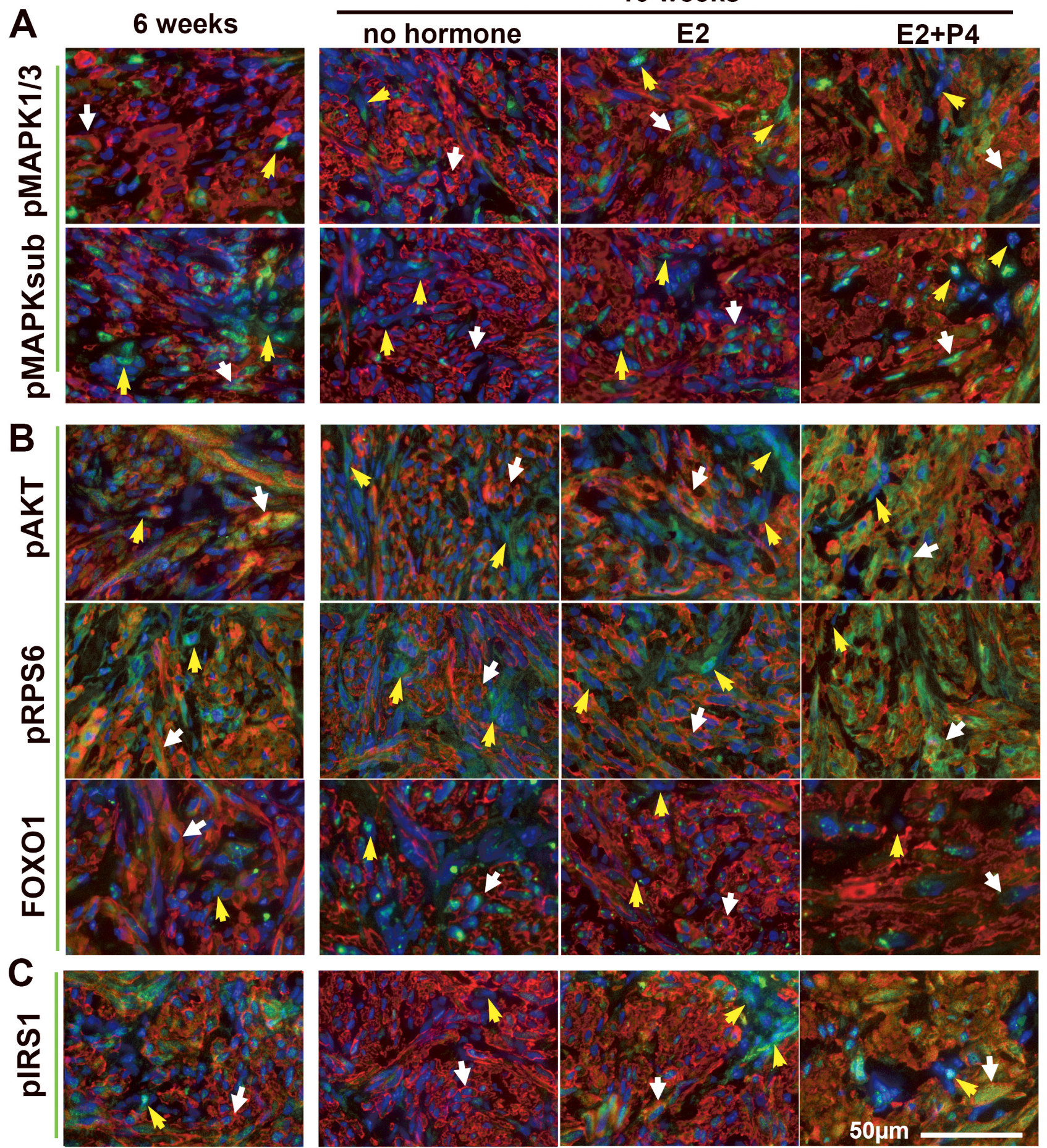

Figure 3

IF analysis on the regulation of signaling pathways by E2 and P4. Following the treatment schedule indicated in Fig. 2A and sampled at the 6- and 10-week timepoints, MAPK, PI3K, and insulin/IGF pathway activities are detected by phosphorylation (p) of target proteins or localization of FOXO1. IF staining of (A) MAPK pathway: PMAPK1/3 and pMAPK substrate (B) PI3K pathway: pAKT, pRPS6, and FOX01 (C) Insulin/IGF pathway: pIRS1(green). In all images, SMCs are highlighted by ACTA2 (red) and nuclei are stained with Hochest (blue). White arrows, SMCs; yellow arrows, TAFs. FOXO1, Forkhead box protein 01; PI3K, phosphoinositide 3-kinase; SMC, smooth muscle cells; TAF, tumor-associated fibroblasts. 
the nuclear accumulation of FOXO1 was attenuated by E2 in both SMCs and TAFs (Fig. 3A and B), confirming that E2 has P4-independent activities in MED12-LMs.

\section{E2 and P4 activate the IGF pathway}

IGFs have been proposed to play critical roles in the growth and survival of LM cells (Gkioka et al. 2015, Moravek et al. 2017). Activation of IGF1R by IGFs induces phosphorylation of insulin receptor substrate 1 (IRS1), and pIRS1 in turn activates several signaling pathways, including the MAPK and the PI3K pathways. Indeed, the levels of pIRS1 parallel with pMAPK1/3 and pAKT, suggesting that the IGF pathway may be the downstream of E2 and P4 in MED12-LMs. In particular, P4-independent action of E2 on TAFs was clearly demonstrated by the upregulation of pIRS1 (Fig. 3C). Accordingly, the expression patterns of IGF ligands (IGF1 and IGF2) and the receptor (IGF1R) within MED12-LM PDXs were examined by IF assay (Fig. 4). In SMCs, IGF1, IGF2 and IGF1R expression increased in response to E2+P4 (Fig. 4A, white arrows). Quantitative IF analysis confirmed this conclusion (Fig. 4B and C). Compared to the NH group, the signal intensity for IGF1, IGF2 and IGF1R in SMCs increased by $15-30 \%$ in the E2+P4 group (Fig. 4B). As cell size also increased with $\mathrm{E} 2+\mathrm{P} 4$, the levels of IGF1, IGF2 and IGF1R per cell was >9-fold higher in the E2+P4-treated groups compared to the other two groups (Fig. 4C).

On the other hand, the expression levels of IGF1, IGF2 and IGF1R were relatively constant in TAFs (Fig. 4A, yellow arrows). In the absence of P4, the signals for IGF1 and IGF1R remained highly concentrated in vascular cells (red arrow heads in Fig. 4A). The localization of IGF1 and IGF1R in endothelial cells suggests that IGF pathway activity may be involved in the maintenance of vascular integrity within MED12-LMs.
A

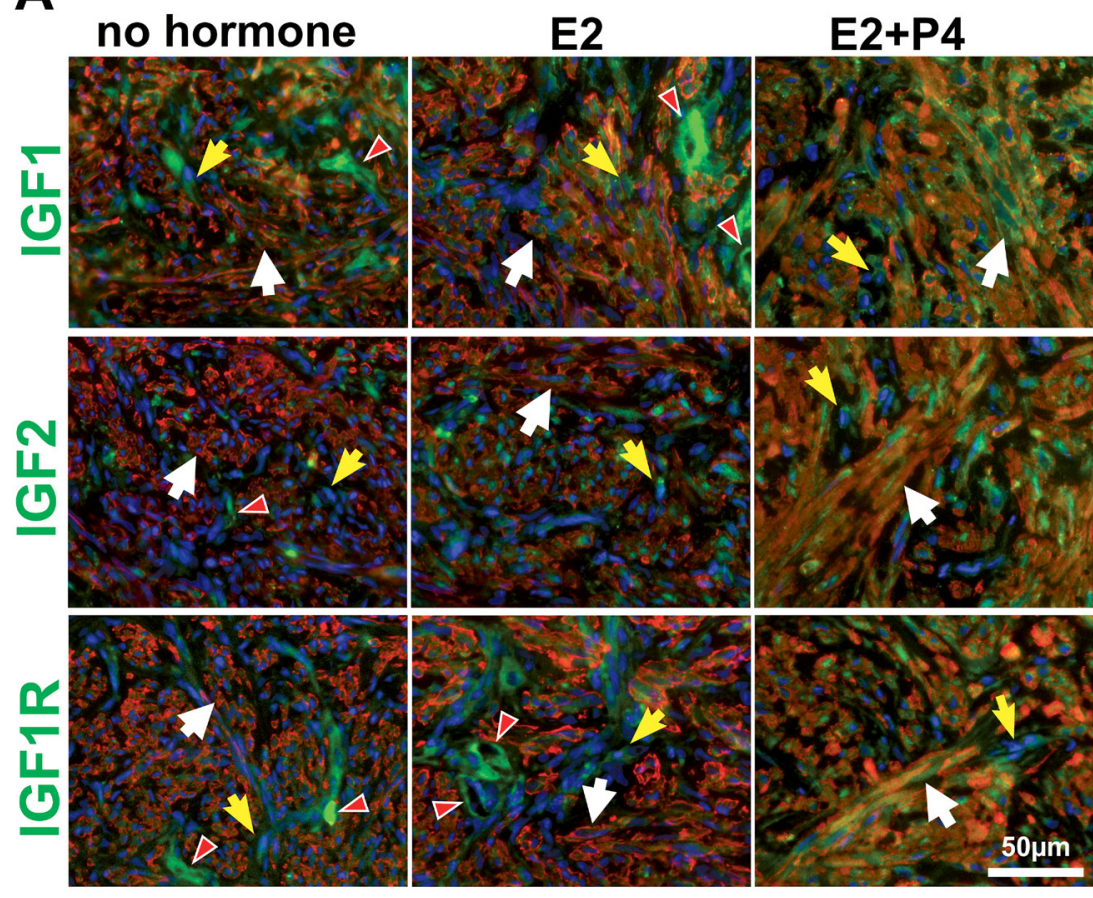

B $\square$ no hormone $\square$ E2 $\square$ E2+P4

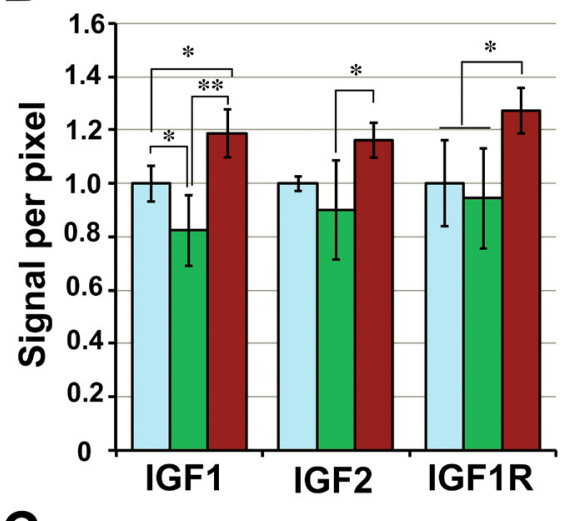

C

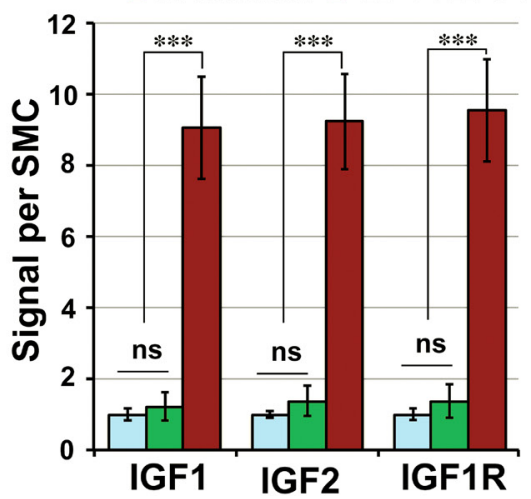

Figure 4

Tissue distribution of IGF pathway molecules. (A) IF analysis of IGF1, IGF2 and IGF1R (green) in PDXs sample at 10-week time point with no hormone, E2 or E2+P4 treatment. In all color images SMCs are highlighted by ACTA2 (red) and nuclei are stained with Hochest (blue). White arrows, SMCs; yellow arrows, TAFs; and red arrowhead, vascular cells. Quantitative analysis of IF signals: the signal density (B) and the total protein level per SMC (C) were measured in PDXs of no hormone $(N=5), E 2(N=4)$ and E2+P4 $(N=6)$ groups. The statistical significance by Tukey's HSD Test was indicated as: ns, non-significant; ${ }^{*} P<0.1 ;{ }^{*} P<0.05$; and ${ }^{* * *} P<0.01$. PDX, patient-derived xenograft; SMC, smooth muscle cells; TAF, tumor-associated fibroblasts. 
Minimum contribution of mouse host vascular cells to MED12-LM PDXs

In general, vasculatures in xenografts originate from mouse hosts. Hence, we examined if vascular cells were host-mouse or human LM in origin, utilizing antiPECAM1 antibodies that preferentially recognize human or mouse endothelial cells (Serna \& Kurita 2018). Since both antibodies cross-react with endothelial cells of both species to some extent, nuclear morphology of cells was also used to identify the species (Kurita et al. 2005): Hoechst nuclear staining highlights heterochromatin foci in mouse cells (Cunha \& Vanderslice 1984). In MED12-LM
PDXs, blood vessels were highlighted by signals for anti-human PECAM1 (Fig. 5A left panel), whereas the anti-mouse PECAM1 antibody stained only a small portion of blood vessels (Fig. 5A right panel). Even then, most endothelial cells showing signals for anti-mouse PECAM1 contained no heterochromatin foci in the nucleus by Hoechst staining (Fig. 5B), indicating their human origin. Hence, the vascular cells in MED12-LM PDXs are mostly derived from original LMs. Accordingly, the high levels of IGF1-IGF1R in the vascular cells of PDXs likely replicates the characteristics of original MED12-LMs in situ. Nonetheless, the contribution of mouse host cells to the PDX was not totally absent: a small number of

\section{A anti-human + anti-mouse}
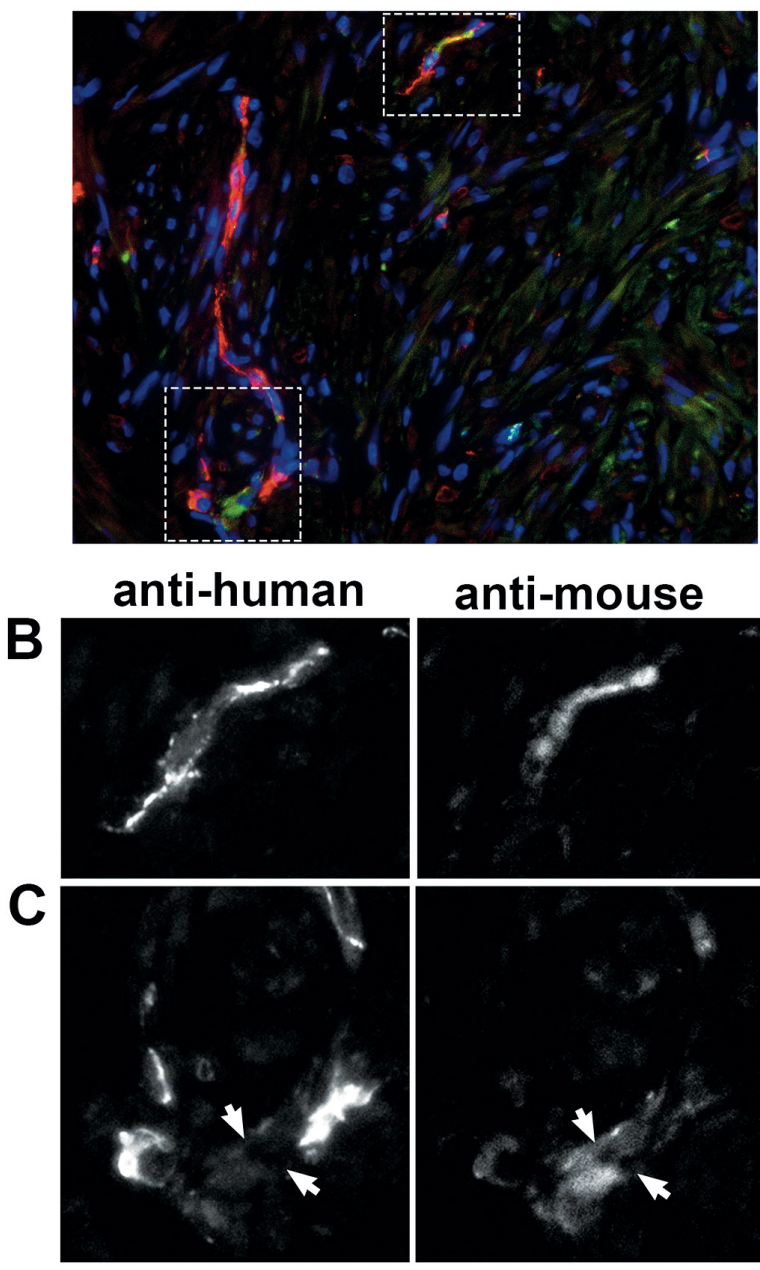

anti-mouse

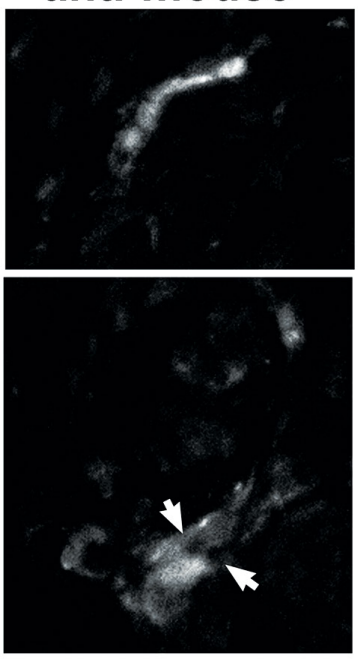

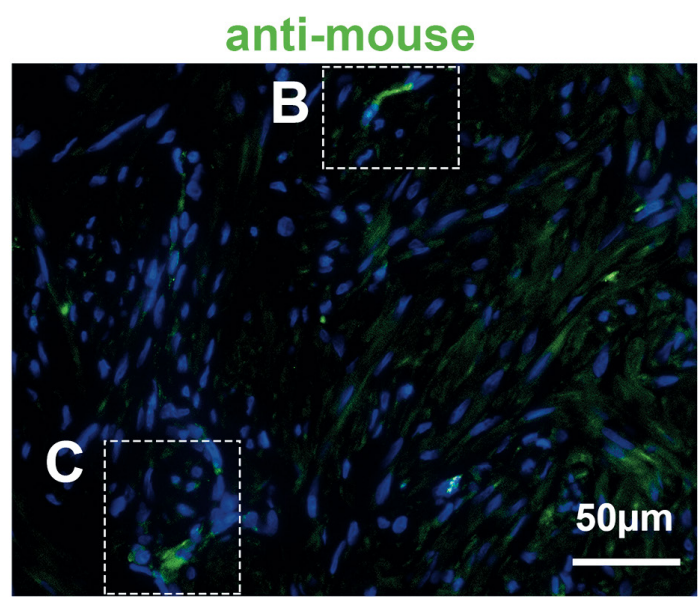

Hochest

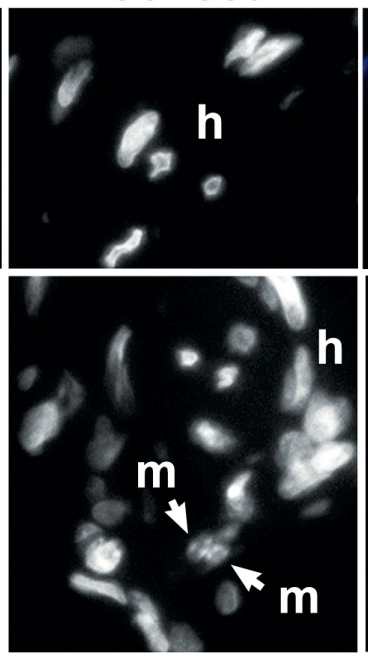

merge

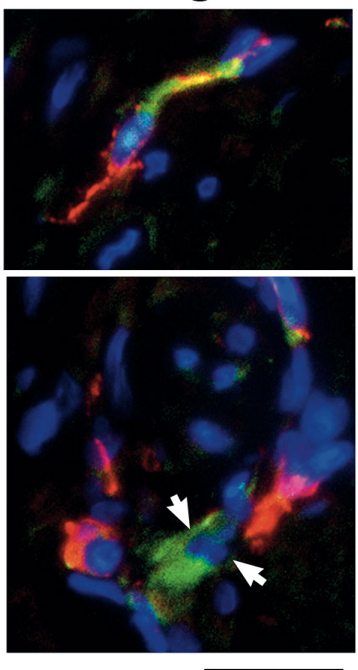

$25 \mu \mathrm{m}$

\section{Figure 5}

The origin of vascular cells in PDX. IF assay for anti-human (red) and anti-mouse (green) PECAM1 antibodies. (A) Macro images. The areas enclosed with a dotted line are magnified in the images below. (Row B) IF assay for each antibody and Hochest staining in separate gray-scale channels and then merged demonstrates the human origin (h) of endothelial cells stained with anti-mouse PECAM1. (Row C) Likewise, a small number of mouse endothelial cells $(\mathrm{m})$ are integrated in vasculature with human endothelial cells (h). PDX, patient-derived xenograft. 
mouse endothelial cells were occasionally found in blood vessels integrated with human endothelial cells (Fig. 5C).

\section{Ultrastructure of ECM in MED12-LM PDXs}

Excess ECM accumulation, especially overexpression of collagens, is a characteristic of MED12-LMs. It has been proposed that accumulation of abnormal collagen fibers drives the pathogenesis of LMs by modulating signal transduction within LM cells (Leppert et al. 2004, Rogers et al. 2008, Koohestani et al. 2013, Jayes et al. 2016). Given E2 and P4 stimulate the production of collagens in LMs (Qiang et al. 2014), we examined the effect of E2 and P4 on the ultrastructure of ECM in MED12-LM PDXs (Fig. 2A) by TEM. Collagen fibers in young-growing MED12-LM
PDXs (4 weeks in Fig. 2A) formed thick and well-aligned bundles (Fig. 6A, D and G). Subsequently, by 10 weeks, collagen fibers remained intact with E2-treatment alone (Fig. 6B, E and $\mathrm{H}$ ); however, the overall electron density of the ECM was significantly reduced in the E2+P4-treated group (Fig. 6C, F and I). Furthermore, collagen fibers in the PDXs of E2+P4 group became fragmented and unaligned, as previously observed in human LM tissues (Leppert et al. 2004, Rogers et al. 2008). This indicates that disruption of collagen fibers occurs only after LMs have reached excessive size. Hence, the abnormal collagen fiber formation in LMs is the effect but not the cause of LM growth. Nevertheless, this does not exclude that the abnormal ECM environment of fully grown LMs positively or negatively affects the growth of LMs.
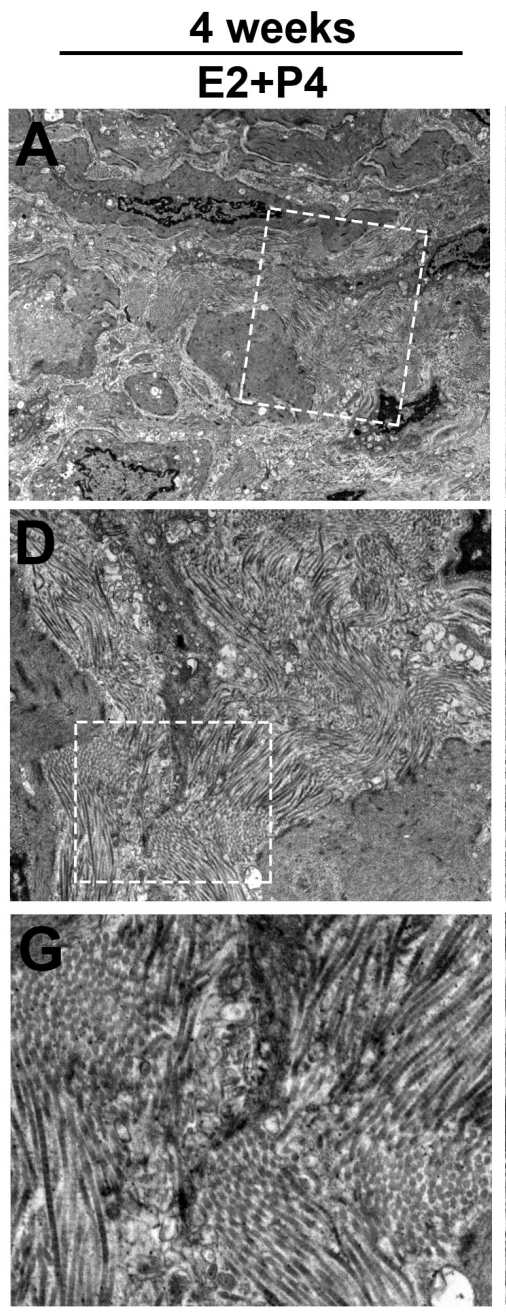
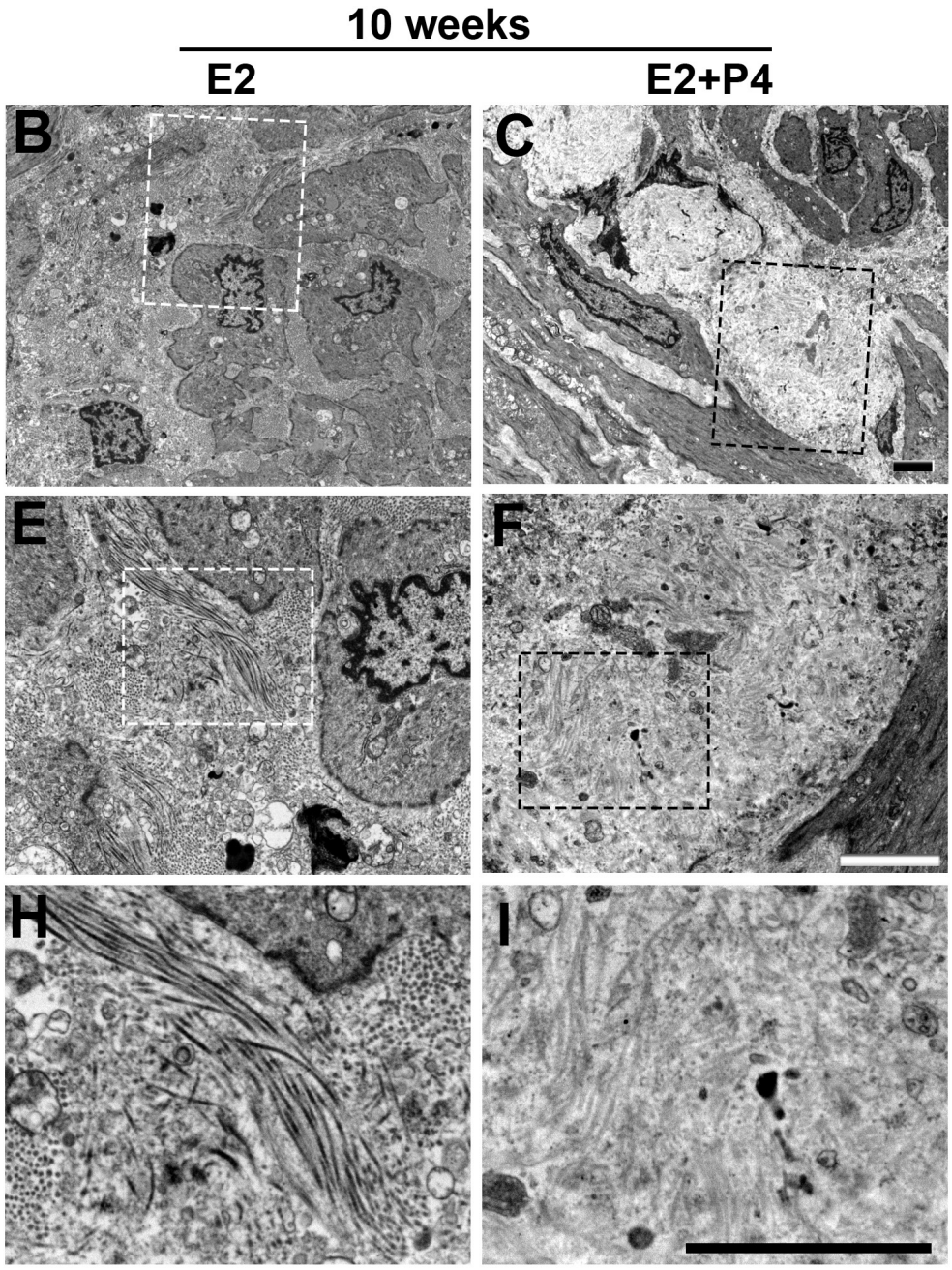

Figure 6

Analysis of collagen fibers in MED12-LMs by TEM. TEM images of MED12-LM PDXs (MED12 C.130G>A): (A, D and G) Early growth at 4 weeks. (B, E and H) Resting at 10 weeks with E2. (C, F and I) Late growth at 10 weeks E2+P4. Images are displayed in three different magnifications (lower to higher from top to bottom) with bars indicating $2 \mu \mathrm{m}$. The area that is magnified in the row below is enclosed with a dotted line. LM, leiomyoma; PDX, patientderived xenograft. 


\section{Discussion}

Signaling pathways involved in the pathogenesis of LMs have been investigated by comparing matched LM and MM tissues. However, the hormonal environment of patients at the time of surgery cannot be precisely defined. Thus, the gene expression profile of LMs may not represent that of the actively growing phase. In addition, LM specimens are usually acquired after LMs have reached a symptomatic size. Hence, some phenotypes of LMs including differential gene expression with MM may be secondary to growth and not the driver of LM pathogenesis. Furthermore, some commonly expressed genes may have different functions in MM and LM. For instance, although ESR1 and PGR are expressed in both LM and MM, only LM tissues grew in response to $\mathrm{E} 2+\mathrm{P} 4$ as xenografts (Ishikawa et al. 2010). For the reasons stated earlier, the signaling pathways involved in the hormone-dependent growth of LMs should be identified through the comparison of LMs exposed to different hormonal environments. For such studies, LM cell cultures have been the primary research model for decades, even though inconsistencies between in vivo and in vitro observations have been long recognized (Severino et al. 1996, Zaitseva et al. 2006). Recent studies identified the root of discrepancies between in vivo and in vitro: primary cultures of MED12LMs consist of primarily TAFs, which do not carry causal mutations of LMs (Markowski et al. 2014, Bloch et al. 2017, Wu et al. 2017). Furthermore, the primary cultures of LMs contain substantial concentrations of endothelial cells, as demonstrated in this study. Additionally, many studies claim the ECM has fundamental effects on the physiology of LM cells (Leppert et al. 2004, Rogers et al. 2008, Koohestani et al. 2013, Jayes et al. 2016). However, the complex structure and biochemistry of natural ECM cannot be replicated in vitro. Accordingly, cell culture is a suboptimal model for LM research. The LM PDX is an ideal alternative, as the IF analysis of the IGF, MAPK and PI3K pathways in this report establishes the efficacy of the PDX in studies of signaling pathways involved in the growth of LMs.

Altered expression of IGF pathway molecules have been described in human LMs (Yu et al. 2008, Baird et al. 2009, Peng et al. 2009). However, the functions of the IGF pathway in the pathogenesis of LMs were studied primarily utilizing in vitro models. Using the MED12-LM PDX model, we determined IGF1 and IGF2 are produced by SMCs in response to E2 and P4. Furthermore, the elevation in IGF1 and IGF2 is accompanied with phosphorylation of IRS1, MAPK1/3 and AKT, suggesting that IGF1/2-IGF1R pathway mediates P4 actions in MED12-LMs. Nevertheless, our current study only demonstrates the correlation between activities of signaling pathways and hormone treatments. Thus, the functional test for the requirement of the IGF-IGF1R pathway in the growth and maintenance of LMs will remain a future study.

In tumor xenograft models, grafted cells/tissues must establish a vascular network with the mouse hosts to survive and grow. In general, blood vessels in tumor xenografts are purely derived from mouse hosts. Accordingly, the ability to recruit host vascular cells is one of primary determinants for successful growth of xenografts (Wang et al. 2016). In contrast, blood vessels in MED12-LM PDXs are primarily derived from original LM tissues, indicating that the initial pool of LM cells contains a substantial concentration of endothelial cells. The absence of mouse endothelial cells within PDXs suggests that the human blood vessels are connected to the host vascular systems around the boundary of PDXs, as LM cells lack the ability to recruit host vasculature cells into the graft. Given the low vascular density of LMs compared to MMs (Casey et al. 2000, Weston et al. 2005), the low angiogenic activity of LM cells is not surprising. As the formation of an intragraft vascular network primarily depends on the endothelial cells derived from the original tumor, LM PDXs work most efficiently with a heterogeneous cell population that includes vascular cells. This is a unique feature of LM PDXs. Accordingly, PDX assays of sorted LM cells require extra caution: The growth rate of a sorted cell fraction highly depends on the concentration of endothelial cells in the population, not necessarily the growth capacity per se.

In this study, we established that loss of LM cells through apoptosis does not play a role in the regression of LMs. However, we acknowledge that the use of the kidney as a blood supply to the PDXs may compromise this determination. The vascular integrity of the kidney is not under the control of steroid hormones, whereas blood flow in MM and endometrium is under the influence of ovarian steroids (Rogers et al. 2009). Hence, the response of LMs to hormones may greatly depend on the location within the uterus, and LM cells may indeed undergo apoptosis in response to hormone withdrawal or antihormone treatment, if such changes compromise the blood supply to the tumor. In addition, the timeframes of our experiments were relatively short ( $\leq 3$ months). Thus, it remains unknown if LM cells can survive without hormones for years (menopause). Notably, IGF1 and IGF1R remain enriched in vascular cells irrespective of hormone treatment. Hence, when systemic levels of E2 
and P4 are low, circulating IGF1 may compensate for the reduced IGF production within the tumor, promoting the survival of LM cells.

The IGF-IGF1R pathway is likely one of many potential mediators of E2 and P4 actions in LMs. Recent comprehensive transcriptome analysis of LM subtypes detected a wide spectrum of genes for growth factors and cytokines (Mehine et al. 2013, 2016). Thus, the hormonal regulation of other signaling pathways in LMs should be studied in LM PDX in the future.

\section{Declaration of interest}

The authors declare that there is no conflict of interest that could be perceived as prejudicing the impartiality of the research reported.

\section{Funding}

This work was supported by the Eunice Kennedy Shriver National Institute of Child Health and Human Development (grant number R01 HD064402) and the National Cancer Institute (grant numbers R01 CA154358 and P30 CA016058).

\section{Author contribution statement}

V A, W Q and T K designed the study. V A, X W, W Q, J T and T K performed the experiments. $M L B$ provided the materials. $V A$ and $T K$ wrote the manuscript. All authors discussed the results and commented on the manuscript.

\section{Acknowledgements}

The authors thank Stacy Ann Kujawa for assistance in patient consent. Sequencing was performed by Northwestern University NuSeq and the OSUCCC-Genomics core. TEM grids were prepared by Lennell Reynolds Jr. at the Northwestern University Center for Advanced Microscopy. TEM images presented in this article were generated using the instruments and services at the Ohio State University Campus Microscopy and Imaging Facility.

\section{References}

Baird DD, Dunson DB, Hill MC, Cousins D \& Schectman JM 2003 High cumulative incidence of uterine leiomyoma in black and white women: ultrasound evidence. American Journal of Obstetrics and Gynecology 188 100-107. (https://doi.org/10.1067/mob.2003.99)

Baird DD, Travlos G, Wilson R, Dunson DB, Hill MC, D'Aloisio AA London SJ \& Schectman JM 2009 Uterine leiomyomata in relation to insulin-like growth factor-I, insulin, and diabetes. Epidemiology 20 604-610. (https://doi.org/10.1097/ EDE.0b013e31819d8d3f)

Bertsch E, Qiang W, Zhang Q, Espona-Fiedler M, Druschitz S, Liu Y, Mittal K, Kong B, Kurita T \& Wei JJ 2014 MED12 and HMGA2 mutations: two independent genetic events in uterine leiomyoma and leiomyosarcoma. Modern Pathology 27 1144-1153. (https://doi. org/10.1038/modpathol.2013.243)

Bloch J, Holzmann C, Koczan D, Helmke BM \& Bullerdiek J 2017 Factors affecting the loss of MED12-mutated leiomyoma cells during in vitro growth. Oncotarget 8 34762-34772. (https://doi.org/10.18632/ oncotarget.16711)

Bulun SE, Moravek MB, Yin P, Ono M, Coon JS, Dyson MT, Navarro A, Marsh EE, Zhao H, Maruyama T, et al. 2015 Uterine leiomyoma stem cells: linking progesterone to growth. Seminars in Reproductive Medicine 33 357-365. (https://doi.org/10.1055/s-0035-1558451)

Casey R, Rogers PA \& Vollenhoven BJ 2000 An immunohistochemical analysis of fibroid vasculature. Human Reproduction 15 1469-1475. (https://doi.org/10.1093/humrep/15.7.1469)

Cunha GR \& Vanderslice KD 1984 Identification in histological sections of species origin of cells from mouse, rat and human. Stain Technology 59 7-12. (https://doi.org/10.3109/10520298409113823)

Gkioka E, Msaouel P, Philippou A, Vlaghogiannis NI, Vogkou CT, Margiolis A \& Koutsilieris M 2015 Review: the role of insulin-like growth factor-1 signaling pathways in uterine leiomyoma. In Vivo 29 637-649.

Hodgson R, Bhave Chittawar P \& Farquhar C 2017 GnRH agonists for uterine fibroids. Cochrane Database of Systematic Reviews $\mathbf{1 0}$ CD012846. (https://doi.org/10.1002/14651858.CD012846)

Holdsworth-Carson SJ, Zhao D, Cann L, Bittinger S, Nowell CJ \& Rogers PA 2016 Differences in the cellular composition of small versus large uterine fibroids. Reproduction 152 467-480. (https://doi. org/10.1530/REP-16-0216)

Ishikawa H, Ishi K, Serna VA, Kakazu R, Bulun SE \& Kurita T 2010 Progesterone is essential for maintenance and growth of uterine leiomyoma. Endocrinology 151 2433-2442. (https://doi.org/10.1210/ en.2009-1225)

Jayes FL, Liu B, Moutos FT, Kuchibhatla M, Guilak F \& Leppert PC 2016 Loss of stiffness in collagen-rich uterine fibroids after digestion with purified collagenase Clostridium histolyticum. American Journal of Obstetrics and Gynecology 215 596.e591-596.e598. (https://doi. org/10.1016/j.ajog.2016.05.006)

Kawaguchi K, Fujii S, Konishi I, Nanbu Y, Nonogaki H \& Mori T 1989 Mitotic activity in uterine leiomyomas during the menstrual cycle. American Journal of Obstetrics and Gynecology 160 637-641. (https:// doi.org/10.1016/S0002-9378(89)80046-8)

Koohestani F, Braundmeier AG, Mahdian A, Seo J, Bi J \& Nowak RA 2013 Extracellular matrix collagen alters cell proliferation and cell cycle progression of human uterine leiomyoma smooth muscle cells. PLOS ONE 8 e75844. (https://doi.org/10.1371/journal.pone.0075844)

Kurita T, Medina R, Schabel AB, Young P, Gama P, Parekh TV, Brody J, Cunha GR, Osteen KG, Bruner-Tran KL, et al. 2005 The activation function-1 domain of estrogen receptor alpha in uterine stromal cells is required for mouse but not human uterine epithelial response to estrogen. Differentiation 73 313-322. (https://doi. org/10.1111/j.1432-0436.2005.00033.x)

Lamminen S, Rantala I, Helin H, Rorarius M \& Tuimala R 1992 Proliferative activity of human uterine leiomyoma cells as measured by automatic image analysis. Gynecologic and Obstetric Investigation 34 111-114. (https://doi.org/10.1159/000292738)

Leppert PC, Baginski T, Prupas C, Catherino WH, Pletcher S \& Segars JH 2004 Comparative ultrastructure of collagen fibrils in uterine leiomyomas and normal myometrium. Fertility and Sterility $\mathbf{8 2}$ (Supplement 3) 1182-1187. (https://doi.org/10.1016/j. fertnstert.2004.04.030)

Mäkinen N, Mehine M, Tolvanen J, Kaasinen E, Li Y, Lehtonen HJ, Gentile M, Yan J, Enge M, Taipale M, et al. 2011 MED12, the mediator complex subunit 12 gene, is mutated at high frequency in uterine leiomyomas. Science 334 252-255. (https://doi.org/10.1126/ science.1208930)

Makinen N, Kampjarvi K, Frizzell N, Butzow R \& Vahteristo P 2017 Characterization of MED12, HMGA2, and FH alterations reveals molecular variability in uterine smooth muscle tumors. Molecular Cancer 16 101. (https://doi.org/10.1186/s12943-017-0672-1)

Markowski DN, Bartnitzke S, Loning T, Drieschner N, Helmke BM \& Bullerdiek J 2012 MED12 mutations in uterine fibroids - their
(2) 2018 Society for Endocrinology Published by Bioscientifica Ltd. Printed in Great Britain 
relationship to cytogenetic subgroups. International Journal of Cancer 131 1528-1536. (https://doi.org/10.1002/ijc.27424)

Markowski DN, Tadayyon M, Bartnitzke S, Belge G, Maria Helmke B \& Bullerdiek J 2014 Cell cultures in uterine leiomyomas: rapid disappearance of cells carrying MED12 mutations. Genes Chromosomes and Cancer 53 317-323. (https://doi.org/10.1002/gcc.22142)

Marshall LM, Spiegelman D, Barbieri RL, Goldman MB, Manson JE, Colditz GA, Willett WC \& Hunter DJ 1997 Variation in the incidence of uterine leiomyoma among premenopausal women by age and race. Obstetrics and Gynecology 90 967-973. (https://doi. org/10.1016/S0029-7844(97)00534-6)

Maruo T, Matsuo H, Samoto T, Shimomura Y, Kurachi O, Gao Z, Wang Y, Spitz IM \& Johansson E 2000 Effects of progesterone on uterine leiomyoma growth and apoptosis. Steroids 65 585-592. (https://doi.org/10.1016/S0039-128X(00)00171-9)

Mehine M, Kaasinen E, Mäkinen N, Katainen R, Kampjarvi K, Pitkanen E, Heinonen HR, Butzow R, Kilpivaara O, Kuosmanen A, et al. 2013 Characterization of uterine leiomyomas by whole-genome sequencing. New England Journal of Medicine 369 43-53. (https://doi. org/10.1056/NEJMoa1302736)

Mehine M, Kaasinen E, Heinonen HR, Mäkinen N, Kampjarvi K, Sarvilinna N, Aavikko M, Vaharautio A, Pasanen A, Butzow R, et al. 2016 Integrated data analysis reveals uterine leiomyoma subtypes with distinct driver pathways and biomarkers. PNAS 113 1315-1320. (https://doi.org/10.1073/pnas.1518752113)

Moravek MB, Yin P, Coon JSt, Ono M, Druschitz SA, Malpani SS, Dyson MT, Rademaker AW, Robins JC, Wei JJ, et al. 2017 Paracrine pathways in uterine leiomyoma stem cells involve insulinlike growth factor 2 and insulin receptor A. Journal of Clinical Endocrinology and Metabolism 102 1588-1595. (https://doi.org/10.1210/jc.2016-3497)

Murji A, Whitaker L, Chow TL \& Sobel ML 2017 Selective progesterone receptor modulators (SPRMs) for uterine fibroids. Cochrane Database of Systematic Reviews 4 CD010770. (https://doi. org/10.1002/14651858.CD010770.pub2)

Myers ER, Goodwin S, Landow W, Mauro M, Peterson E, Pron G, Spies JB, Worthington-Kirsch R \& Investigators F 2005 Prospective data collection of a new procedure by a specialty society: the FIBROID registry. Obstetrics and Gynecology 106 44-51. (https://doi. org/10.1097/01.AOG.0000165827.66541.dd)

Palomba S, Sena T, Noia R, Di Carlo C, Zullo F \& Mastrantonio P 2001 Transdermal hormone replacement therapy in postmenopausal women with uterine leiomyomas. Obstetrics and Gynecology $\mathbf{9 8}$ 1053-1058. (https://doi.org/10.1016/S0029-7844(01)01598-8)

Parker WH 2007 Etiology, symptomatology, and diagnosis of uterine myomas. Fertility and Sterility 87 725-736. (https://doi.org/10.1016/j. fertnstert.2007.01.093)

Peng L, Wen Y, Han Y, Wei A, Shi G, Mizuguchi M, Lee P, Hernando E, Mittal K \& Wei JJ 2009 Expression of insulin-like growth factors (IGFs) and IGF signaling: molecular complexity in uterine leiomyomas. Fertility and Sterility 91 2664-2675. (https://doi. org/10.1016/j.fertnstert.2007.10.083)

Qiang W, Liu Z, Serna VA, Druschitz SA, Liu Y, Espona-Fiedler M, Wei JJ \& Kurita T 2014 Down-regulation of miR-29b is essential for pathogenesis of uterine leiomyoma. Endocrinology 155 663-669. (https://doi.org/10.1210/en.2013-1763)

Rogers R, Norian J, Malik M, Christman G, Abu-Asab M, Chen F, Korecki C, Iatridis J, Catherino WH, Tuan RS, et al. 2008 Mechanical homeostasis is altered in uterine leiomyoma. American Journal of Obstetrics and Gynecology 198 e471-e411. (https://doi.org/10.1016/j. ajog.2007.06.018)

Rogers PA, Donoghue JF, Walter LM \& Girling JE 2009 Endometrial angiogenesis, vascular maturation, and lymphangiogenesis. Reproductive Sciences 16 147-151. (https://doi. org/10.1177/1933719108325509)

Sener AB, Seckin NC, Ozmen S, Gokmen O, Dogu N \& Ekici E 1996 The effects of hormone replacement therapy on uterine fibroids in postmenopausal women. Fertility and Sterility $65354-357$. (https:// doi.org/10.1016/S0015-0282(16)58098-4)

Serna VA \& Kurita T 2018 Patient-derived xenograft model for uterine leiomyoma by sub-renal capsule grafting. Journal of Biological Methods 5 e91. (https://doi.org/10.14440/jbm.2018.243)

Severino MF, Murray MJ, Brandon DD, Clinton GM, Burry KA \& Novy MJ 1996 Rapid loss of oestrogen and progesterone receptors in human leiomyoma and myometrial explant cultures. Molecular Human Reproduction 2 823-828. (https://doi.org/10.1093/ molehr/2.11.823)

Terakawa J, Rocchi A, Serna VA, Bottinger EP, Graff JM \& Kurita T 2016 FGFR2IIIb-MAPK activity is required for epithelial cell fate decision in the lower mullerian duct. Molecular Endocrinology 30 783-795. (https://doi.org/10.1210/me.2016-1027)

Tiltman AJ 1985 The effect of progestins on the mitotic activity of uterine fibromyomas. International Journal of Gynecological Pathology 4 89-96. (https://doi.org/10.1097/00004347-198506000-00001)

Wang G, Ishikawa H, Sone K, Kobayashi T, Kim JJ, Kurita T \& Shozu M 2014 Nonobese diabetic/severe combined immunodeficient murine xenograft model for human uterine leiomyoma. Fertility and Sterility 101 1485-1492. (https://doi. org/10.1016/j.fertnstert.2014.01.054)

Wang Y, Wang JX, Xue H, Lin D, Dong X, Gout PW, Gao X \& Pang J 2016 Subrenal capsule grafting technology in human cancer modeling and translational cancer research. Differentiation 91 15-19. (https://doi.org/10.1016/j.diff.2015.10.012)

Weston GC, Cattrall F, Lederman F, Vollenhoven BJ \& Rogers PA 2005 Differences between the pre-menopausal and post-menopausal uterine fibroid vasculature. Maturitas 51 343-348. (https://doi. org/10.1016/j.maturitas.2004.08.017)

Wu X, Serna VA, Thomas J, Qiang W, Blumenfeld ML \& Kurita T 2017 Subtype-specific tumor-associated fibroblasts contribute to the pathogenesis of uterine leiomyoma. Cancer Research 77 6891-6901. (https://doi.org/10.1158/0008-5472.CAN-17-1744)

Yang CH, Lee JN, Hsu SC, Kuo CH \& Tsai EM 2002 Effect of hormone replacement therapy on uterine fibroids in postmenopausal women - a 3-year study. Maturitas 43 35-39. (https://doi.org/10.1016/S03785122(02)00159-7)

Yu L, Saile K, Swartz CD, He H, Zheng X, Kissling GE, Di X, Lucas S, Robboy SJ \& Dixon D 2008 Differential expression of receptor tyrosine kinases (RTKs) and IGF-I pathway activation in human uterine leiomyomas. Molecular Medicine 14 264-275. (https://doi. org/10.2119/2007-00101.Yu)

Zaitseva M, Vollenhoven BJ \& Rogers PA 2006 In vitro culture significantly alters gene expression profiles and reduces differences between myometrial and fibroid smooth muscle cells. Molecular Human Reproduction 12 187-207. (https://doi.org/10.1093/molehr/ gal018)

Received in final form 6 April 2018

Accepted 25 April 2018

Accepted Preprint published online 26 April 2018 (c) 2018 Society for Endocrinology Published by Bioscientifica Ltd. Printed in Great Britain 